\title{
UNA ADORACIÓN DE LOS MAGOS FIRMADA POR ANTONIO CASTEJÓN: NUEVA INCORPORACIÓN A SU CATÁLOGO
}

\author{
JoAQuín SÁez Vidal \\ Doctor en Historia del Arte
}

\begin{abstract}
El autor estudia y da a conocer una obra desconocida del pintor barroco español Antonio Castejón (h. 1634-1696), lienzo que se conserva en la basílica de Santa María de Elche (Alicante).

Palabras clave: Pintura española; Barroco; Escuela de Madrid; Siglo XVII; Antonio Castejón; Elche.
\end{abstract}

\section{AN ADORATION OF THE MAGI SIGNED BY ANTONIO CASTEJÓN: A NEW ADDITION TO THE CATALOG OF HIS WORK}

The author presents and analizes a new work by the Spanish Baroque painter Antonio Castejón (c. 1634-1696). This unpublished canvas is preserved in the basilica of Santa Maria in Elche (Alicante).

Key words: Spanish Painting; Baroque; School of Madrid; $17^{\text {th }}$ Century; Antonio Castejón; Elche.

Dentro de la amplia nómina de pintores pertenecientes a la escuela madrileña de la segunda mitad del siglo XVII, sin la reputación de Carreño, Coello, Rizi y algún otro, hay que situar la figura de Antonio Castejón (h. 1634-1696). Su labor como pintor resulta todavía muy mal conocida, siendo apenas mencionado, y en general poco valorado, en la bibliografía artística desde que Palomino lo incluyera en su famoso tratado. Por lo que éste nos relata, Castejón fue un «pintor práctico; y aunque amanerado tuvo gran facilidad de invención ${ }^{1} »$. Su estilo, especialmente en lo que denomina «historiejas pequeñas» lo enmarca en la órbita de pintores como Roque Ponce, José García Hidalgo y Gabriel de la Corte. También se ocupó de composiciones de gran tamaño de carácter religioso que sitúa en numerosas iglesias de Madrid, como las de San Miguel, San Felipe, San Ginés, el Carmen Calzado y la Virgen de Gracia, obras que en su mayoría se han destruido o desaparecido. Además de lienzos de dicha temática, hay constancia de su actividad como retratista e incluso, según sabemos por Mercedes Agulló, autor de mitologías². Poca información añaden en sus tratados Antonio Ponz y Ceán Bermúdez al referirse a nuestro pintor. Sin embargo, durante los últimos años han aparecido importantes artículos sobre nuevos hallazgos de pinturas, tanto de asunto religioso como retratos, que amplían el conocimiento de su todavía escasísima obra conservada.

En efecto, de lo que debió de ser una amplia producción, en la actualidad el catálogo de sus pinturas recoge apenas una decena. Entre las piezas recientemente localizadas cabe señalar una Inmaculada Concepción dada a conocer por $\mathrm{M}^{\mathrm{a}}$ Socorro Salvador que se encuentra en la iglesia de Rebollosa de Hita, Guadalajara ${ }^{3}$. De dimensiones considerables y en deficiente estado de con-

\footnotetext{
${ }^{1}$ Palomino y Velasco, 1947, p. 1048.

2 Agulló Cobo, 1981, p. 220.

${ }^{3}$ Salvador Prieto, 1991, pp.289-294. El lienzo mide 180 x $102 \mathrm{~cm}$.
} 
servación, lleva la firma de "ANTONIO CASTREJÓN" en el ángulo inferior izquierdo. En su factura se advierte una estrecha vinculación, al igual que sucede con otros lienzos suyos, con los modelos del círculo artístico de Carreño de Miranda ${ }^{4}$.

Este mismo motivo iconográfico, tan familiar en la pintura española del Barroco, lo representa nuestro pintor en otro lienzo, de tamaño ligeramente mayor que el anterior $\left(189 \times 136^{\prime} 5 \mathrm{~cm}\right)$, que se encuentra en el Colegio Mayor "Ximénez de Cisneros" de la Ciudad Universitaria de Madrid. El hallazgo y estudio de la obra, que había pasado completamente desapercibida pese a figurar la firma del artista si bien no está fechada, se debe a Portela Sandoval ${ }^{5}$, quien señala que la imagen mariana está igualmente "en la línea de las inmaculadas de Carreño". El repertorio mariano de Castejón se ampliaba con otras versiones de la Inmaculada Concepción, como la que Diego Angulo menciona en uno de sus libros, aunque sin reproducirla, al parecer firmada en 1690. Dicho ejemplar, de acuerdo con lo afirmado por él, se encontraba en la iglesia del Carmen Calzado de Madrid, si bien se desconoce su paradero actual ${ }^{7}$.

Distinta iconografía, aunque igualmente de temática religiosa, resulta el lienzo de la Huída a Egipto, firmado pero no fechado, que se conserva en el convento de las Úrsulas de Alcalá de Henares ${ }^{8}$. En opinión de Gutiérrez Pastor, "con figuras de canon corto y formas anchas, acordes con obras de otros contemporáneos de Castrejón, como Diego González de Vega o José García Hidalgo"". En un trabajo posterior, Gutiérrez Pastor encuentra no pocos puntos en común entre esta obra y los dos ejemplares del mismo asunto del pintor José Moreno, uno conservado en el Museo del Pardo y el otro en el Minneapolis Institute of $\mathrm{Art}^{10}$, este último fechado, según información del propio museo, en $1669^{11}$. Una segunda versión de la Huída a Egipto de Castejón, también con evidentes analogías formales con los modelos de José Moreno ${ }^{12}$, así como otras dos que representan El descanso en la huída a Egipto y La Sagrada Familia, pueden contemplarse en el Hospital madrileño del Niño Jesús ${ }^{13}$, cuadros que cuelgan de una pared alta de una de las cajas de escaleras de dicho hospital. A estas obras hay que añadir un retrato de Doña María Josefa Antonia Rodríguez

${ }^{4}$ Salvador Prieto, 1991, p. 292. En coincidencia con dicha autora, Pérez Sánchez opina que el modelo de Inmaculada dado a conocer por Salvador Prieto, que erróneamente localiza en colección particular madrileña, obliga a situar a Castrejón "en el círculo de Carreño, cuyo modelo copia con algunas variantes". Vid. Pérez Sánchez, 2010, p. 335.

${ }^{5}$ Portela Sandoval, 1995, pp. 561-563. En este lienzo el pintor firma en letras minúsculas "Antonio Castejón” y no "Antonio Castrejon", en letras capitales, como en el cuadro de Rebollosa de Hita.

${ }^{6}$ Portela Sandoval, 1995, p. 563.

7 Angulo Iñiguez, 1971, p. 305. Además de la citada Concepción, Angulo da a conocer otras obras. "Algunas de sus principales pinturas -afirma- son: una Santa Lucía en San Felipe, las historias de la Vida de la Virgen, de la iglesia de San Ginés, el San Pedro del Hospital de los Italianos firmado". Cf. Ibidem.

${ }^{8}$ Caballero Bernabé, Sánchez Galindo y otros, 1990, pp. 307 y 320.

${ }_{9}^{9}$ Gutiérrez Pastor, 1991, p.104. La pintura mide 148 x $210 \mathrm{~cm}$, y aparece firmado únicamente con el apellido "Castexon".

${ }^{10} \mathrm{Al}$ establecer la comparación entre el ejemplar del convento de las Úrsulas de Alcalá de Henares y las obras de idéntico asunto de José Moreno, Gutiérrez Pastor afirma que "aunque existen variaciones, las deudas compositivas (de Castejón) respecto a Moreno son muy estrechas y se relacionan con el modelo de Minneapolis... especialmente la figura del ángel y de San José”. Vid. Gutiérrez Pastor, 2005, p.76.

${ }^{11}$ Sin embargo, en la firma que aparece en el cuadro, sólo se dice "Joseph Moreno F 16..", faltando los dos últimos dígitos. En opinión de Edward J. Sullivan debe corresponder al año 1662. Véase Sullivan y Mallory, 1982, p. 83.

${ }^{12}$ Este segundo ejemplar de La Huída a Egipto del Hospital del Niño Jesús tiene, igualmente, mucho que ver con el lienzo del mismo título del pintor José Moreno perteneciente al Minneapolis Institute of Arts, lo que habla de una relación directa entre ambos artistas. Coincidimos por tanto con lo manifestado con Gutiérrez Pastor cuando afirma que "Las numerosas interpretaciones que Moreno realizó de la Huía a Egipto parecen haberlo convertido en un especialista en el tema y haber sido ejemplo o haber marcado una cierta influencia en otros pintores contemporáneos". Véase Gutiérrez Pastor, 2005, p. 75.

${ }_{13}$ De las tres pinturas de Antonio Castejón existentes en dicho Hospital madrileño, sólo hemos podido localizar dos de ellos, los que representan el Descanso en la huída a Egipto y La huída a Egipto, ignorando el paradero del tercero

Arch. esp. arte, LXXXVIII, 350, ABRIL-JUNIO 2015, 198-204

ISSN: 0004-0428, eISSN: 1988-8511, doi: 10.3989/aearte.2015.13 
de los Ríos y Bueno, hija del I Marqués de Santiago, hallado por Gutiérrez Pastor en el convento madrileño de las MM. Mercedarias de D. Juan de Alarcón ${ }^{14}$, en el que la retratada ingresó a la edad de 14 años. El conjunto de obras conservadas se completaba hasta ahora con una Asunción en el convento de los franciscanos de Olite (Navarra) y una Anunciación dada a conocer también por Gutiérrez Pastor de propiedad particular madrileña, obra esta última que revela, una vez más, como acierta a señalar su descubridor, un estrecho contacto con los modelos del pintor José Moreno ${ }^{15}$.

A este reducido conjunto de pinturas de Castejón hay que añadir una nueva que aquí presentamos por vez primera y que, en nuestra opinión, constituye una de sus creaciones más relevantes. Nos referimos a la Adoración de los Magos que puede contemplarse en la basílica de Santa María de Elche (Alicante), composición que como la mayoría de las suyas resulta de amplio formato, siendo sus medidas $160 \times 238 \mathrm{~cm}$. Con tal aportación, es claro, se incrementa el todavía reducido catálogo de sus obras firmemente atribuidas, permitiendo, además, un mejor conocimiento y valoración de su personalidad artística (fig. 1).

En el lienzo objeto de nuestro estudio el pintor organiza la composición, desarrollada horizontalmente, en dos zonas claramente diferenciadas, siendo la principal la formada por el episodio bíblico que da título al cuadro. Así, en un espacio más próximo al espectador y a mayor tamaño, Castejón sitúa la escena del grupo de los Reyes Magos que presentan sus ofrendas a Jesús niño. Los tres personajes regios ataviados con ropas ricamente bordadas así como otros objetos lujosos que en conjunto ofrecen una nota de gran fastuosidad. Los rostros resultan naturales aunque ligeramente idealizados, con perfiles un tanto afilados. Nuestro pintor, sensible al espíritu barroco al que pertenece, se vale de los típicos contrastes de luces y sombras para dar mayor corporeidad a las tres figuras. En su tratamiento, Castejón, al igual que otros pintores españoles del siglo XVII, parece combinar ecos evidentes de raíz veneciana con préstamos de la pintura flamenca del siglo XVII, elementos perceptibles igualmente en otras obras suyas.

Con la incorporación de los tres actores sacros, en el extremo derecho del cuadro, se completa la iconografía principal del mismo. El grupo de la Sagrada Familia lo ha situado el pintor en un escorzado escenario arquitectónico en el que podemos vislumbrar grandiosas columnas de porte clasicista que se elevan sobre altos basamentos. Es este un recurso ampliamente utilizado por numerosos pintores españoles del barroco. Así, por lo que respecta en particular a la escuela madrileña de la segunda mitad del siglo XVII a la que pertenece Antonio Castejón, pintores como Carreño de Miranda ya habían incluido tal escenografía arquitectónica en, por ejemplo, la Adoración de los Magos de la colección Várez Fisa, al igual que en los Desposorios de Santa Catalina del mismo autor perteneciente a la Venerable Orden Tercera de Madrid ${ }^{16}$. Otro tanto sucede con la obra del mismo título que Mateo Cerezo realiza en 1660 y conservada en el Museo del Prado. Por su parte, Francisco Solís la incluye asimismo en su Anunciación de colección privada madrileña ${ }^{17}$. También figura, por último, y sin pretender ser exhaustivo, en las dos Adoración de los Magos de Francisco Rizi, tanto la del Museo del Prado como la de la catedral de Toledo, o en la que firma José Moreno en 1660 de colección particular, así como en dos lienzos con el tema de la Anunciación de este último pintor conservados en las Salesas de Madrid y en el Williams College de Williamstone, Massachussets.

dedicado a La Sagrada Familia. De dimensiones considerables, como casi todos los suyos, pero sin poder precisar las medidas por la dificultad de su ubicación, ambos están firmados y fechados. El primero de los cuadros está firmado y fechado de la siguiente manera: "ANT CASTEJON FAC. Anno 169(4?)". Por lo que respecta al segundo lienzo lo firma de igual manera pero lo data, según nos parece leer, en 1695. Teniendo en cuenta que su muerte acaeció en 1696, se trataría, pues, de algunas de sus últimas obras.

${ }^{14}$ Gutiérrez Pastor, 1991, p. 104.

${ }^{15}$ Gutiérrez Pastor, 2005, pp. 76 y 78, fig. 13. Se trata de un óleo sobre lienzo de 104 x 205 cm. Firmado "Castexon".

${ }^{16}$ Las fotografías de ambos lienzos figuran en Pérez Sánchez, 1986, pp.114 y 118.

17 El cuadro en cuestión viene reproducido en el artículo de Gutiérrez Pastor, 2000, fig. 21, p. 88.

Arch. esp. arte, LXXXVIII, 350, ABRIL-JUNIO 2015, 198-204 ISSN: 0004-0428, eISSN: 1988-8511, doi: 10.3989/aearte.2015.13 


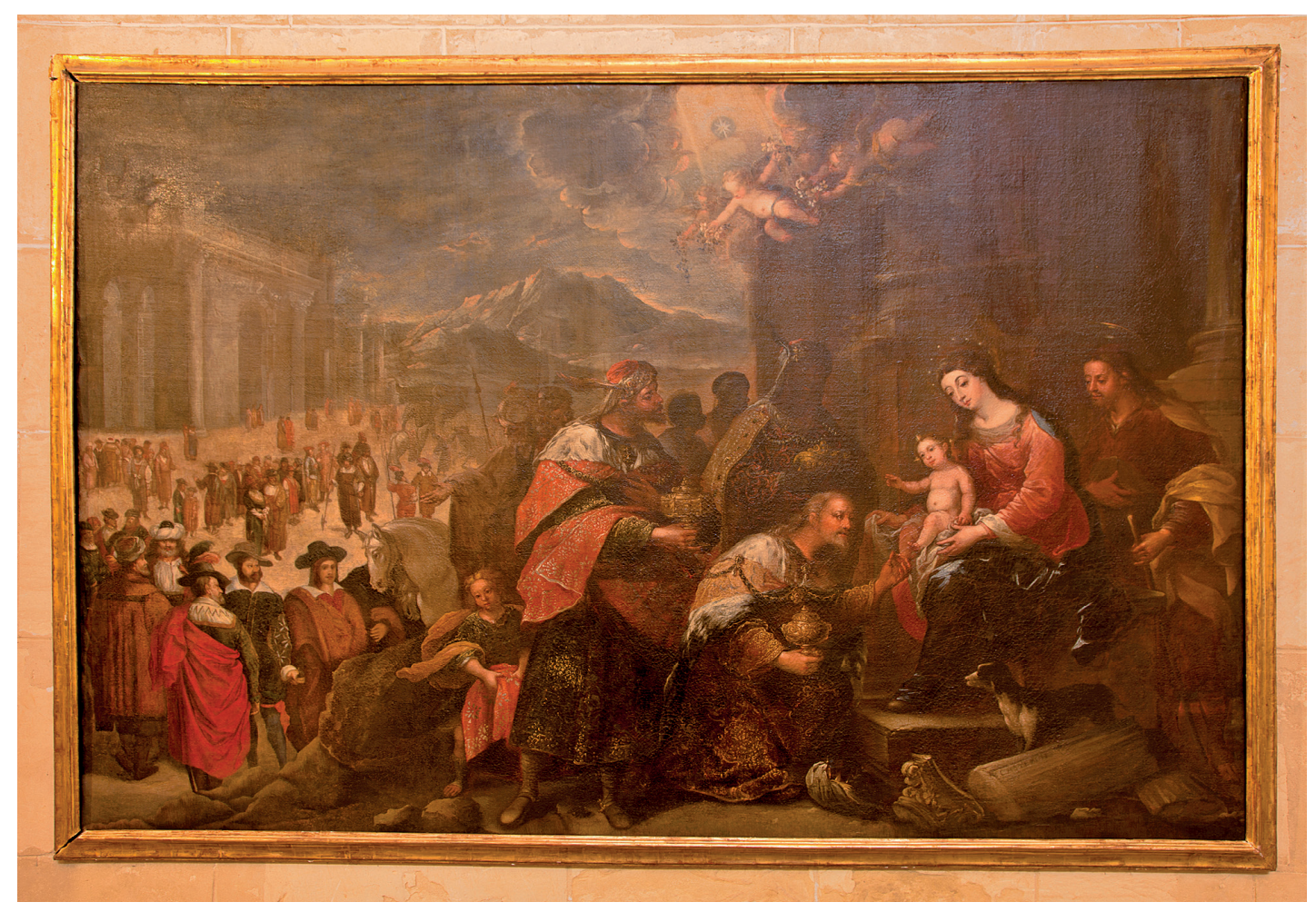

Fig. 1. Antonio Castejón. Adoración de los Magos. Elche, basílica de Santa María.

De las tres figuras sacras destaca por su monumentalidad la imagen de la Virgen, que viste la tradicional túnica rosa y manto azul de brillo tornasolado de sabor veneciano. El rostro, de colorido nacarado, responde a la misma tipología fisonómica de otras vírgenes suyas, caracterizada por su forma ovalada, nariz fina y apuntada -en la línea de Carreño-, aspecto candoroso y belleza dulcificada, modelo que, entre otros, se aproxima a la que figura en la Adoración de los Magos de Francisco Rizi depositada por el Museo del Prado en la Universidad de Barcelona. Un nimbo estrellado rodea su cabeza cubierta por una abundante cabellera cuyos extremos caen sobre los hombros terminando en ondulantes rizos; detalle este último que Castejón también hace figurar en la Virgen de la Anunciación, lienzo, como ya se ha dicho, hoy en propiedad particular madrileña ${ }^{18}$. El cuerpo desnudo del Niño destaca por la fuerte luminosidad, al tiempo que constituye el centro de atención de las miradas de todos los personajes principales del lienzo. Con la figura de San José, que Castejón desplaza al extremo derecho del cuadro, se completa el conjunto de la Sagrada Familia.

Por encima del grupo principal, unos ángeles niños suspendidos en el aire presentan unas ofrendas de flores. Las mismas tipologías infantiles las repite en su ya citado cuadro de la Huída a Egipto del madrileño Hospital del Niño Jesús. En su morfología advertimos una clara inspiración de Castejón en obras de José Moreno, tal como podemos apreciar en los angelitos que aparecen en las pinturas de idéntico asunto de este último artista conservadas una de ellas en el Museo del Prado y la otra en el Minneapolis Institute of Art.

\footnotetext{
${ }^{18}$ Véase en Gutiérrez Pastor, 2005, p.78, fig. 13.
}

Arch. esp. arte, LXXXVIII, 350, ABRIL-JUNIO 2015, 198-204

ISSN: 0004-0428, eISSN: 1988-8511, doi: 10.3989/aearte.2015.13 


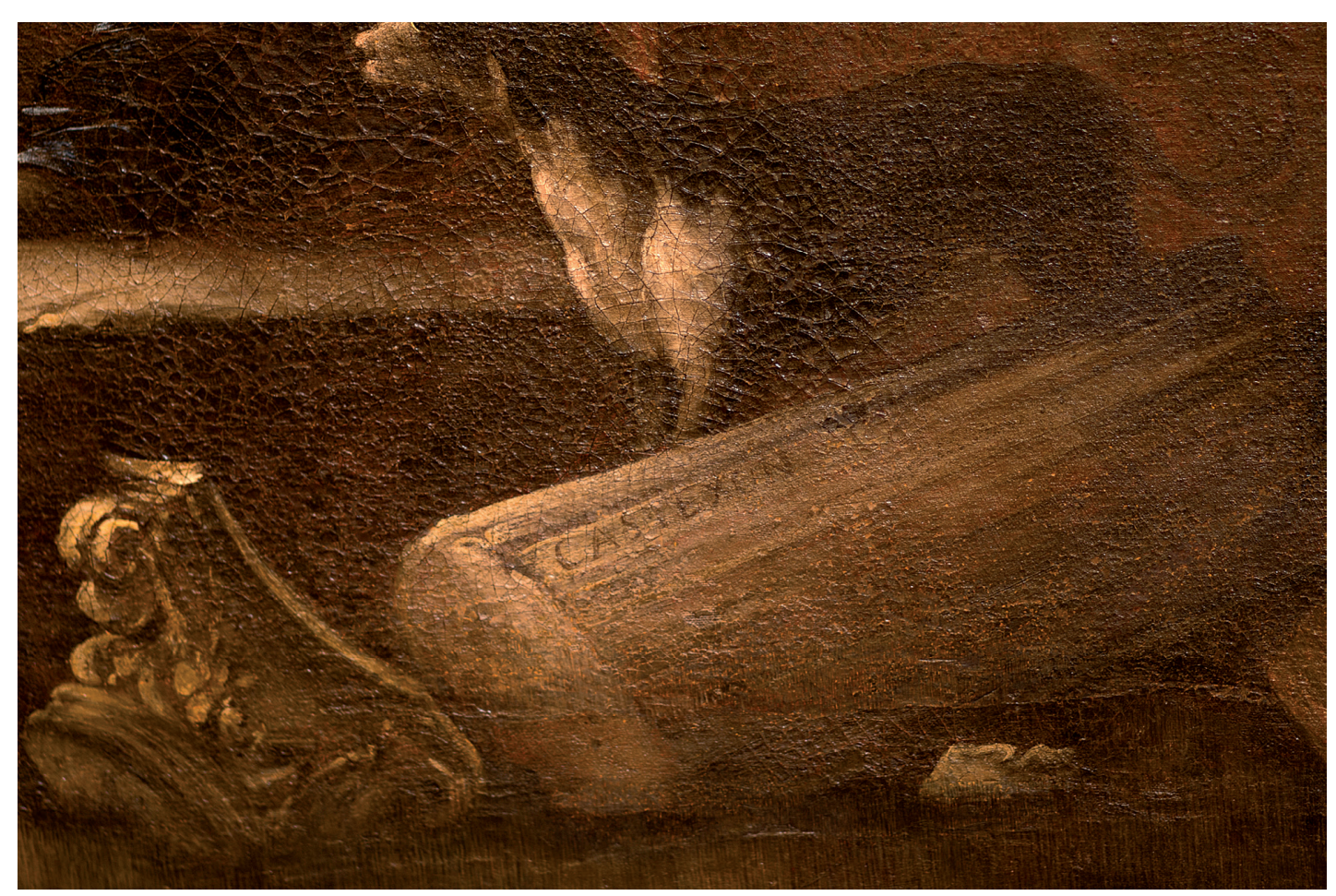

Fig. 2. Antonio Castejón. Adoración de los Magos. Elche, basílica de Santa María. Detalle. (Fotografía del autor).

También resulta de interés por su evidente naturalismo la inclusión de un perro de formas bien definidas que dirige su mirada atenta al grupo de los Magos. Junto al animal Castejón ha dispuesto varias piezas arquitectónicas, como un capitel caído y fragmentos de columnas, todos ellos de inspiración clásica. Con tal incorporación, nuestro pintor, además de añadir una serie de elementos aparentemente anecdóticos a la composición, es posible que haya pretendido evocar, en clave simbólica, la idea del triunfo del cristianismo sobre el paganismo. Una vez más estos detalles muestran una estrecha vinculación con los que aparecen en la Adoración de los Magos de José Moreno, de colección particular, composición que éste realiza en $1660^{19}$. Ello nos hace sospechar que la obra de José Moreno tal vez pudo servir de inspiración a la de Castejón. Junto al capitel de orden corintio, a su derecha, Castejón ha colocado un tambor de columna cuyo mayor interés reside en que allí figura la firma del artista en letras capitales: "CASTEXON" (fig. 2).

Aunque él emplea varias modalidades para firmar sus $\operatorname{cuadros}^{20}$, sólo lo hace exclusivamente con su apellido y en mayúsculas hasta ahora en dos únicos cuadros que firma como en el que es objeto de nuestro estudio, es decir, "CASTEXON". Uno de ellos es la Huída a Egipto, que guarda el convento de las Úrsulas de Alcalá de Henares; el otro es la Anunciación, de colección particular madrileña. Tal circunstancia, pese a no estar datados, nos hace suponer que la realización del cuadro de la basílica de Elche y los otros dos a los que acabamos de referirnos estarían próximos en el tiempo.

${ }^{19}$ Gutiérrez Pastor, 2005, p.71, fig.2.

${ }^{20}$ Así, por ejemplo, en la Inmaculada de Rebollosa de Hita firma en letras capitales "Antonio Castrejon"; en la Inmaculada Concepción existente en el madrileño Colegio Mayor "Ximénez de Cisneros" de la Ciudad Universitaria lo hace como "Antonio Castejón", sin la letra erre; incluso con el nombre abreviado y en mayúsculas, "Ant. Castrejon" aparece en los dos lienzos que hemos podido ver en el Hospital del Niño Jesús de Madrid.

Arch. esp. arte, LXXXVIII, 350, ABRIL-JUNIO 2015, 198-204 ISSN: 0004-0428, eISSN: 1988-8511, doi: 10.3989/aearte.2015.13 
La Adoración de los Magos de Elche presenta, además, en gran parte de la mitad izquierda del lienzo, una escena al aire libre que no deja de parecer un tanto extraña y de cierto sabor manierista. En ella Castejón incorpora un número considerable de figuras que visten a la moda del siglo XVII y que se disponen en un plano más alejado de la escena principal, ajenos, aparentemente, al hecho religioso que allí acontece. En su composición tales personajes aparecen tratados de manera sumaria, sin el cuidado mostrado en la representación de la Sagrada Familia y los tres Reyes.

En este amplio espacio abierto nuestro pintor ha incluido una serie de edificios escorzados de aspecto abocetado a los que dota de un aire a la vez idealizado y misterioso, rasgo que exhala un característico sabor veneciano, desde luego más próximo a la sensibilidad de Tintoretto que a la de Tiziano. La vinculación con dicha escuela, caracterizada por una intensa tonalidad cromática, también puede establecerse en detalles como el fondo montañoso y el cielo azulado. Ello no constituye ninguna originalidad por parte de nuestro pintor, pues es sabido que resulta una tradición muy común en los pintores españoles de escuela madrileña de la segunda mitad del siglo XVII. Formado Castejón en dicha escuela es lógico pensar que él tomaría los componentes flamencos y neovenecianos que son consustanciales a ella.

La obra que acabamos de analizar, ya para finalizar, permite ahondar en un mejor conocimiento, bien es verdad que todavía incompleto, de uno de los más interesantes representantes de la pintura madrileña de la segunda mitad del siglo XVII. Esta nueva Adoración de los Magos de la basílica ilicitana de Santa María no cierra, con todo, los numerosos problemas que todavía plantea la figura de Antonio Castejón, referidos especialmente a cuestiones tales como su proceso formativo y posible evolución de su estilo. Sea lo que fuere, y aunque en otras obras suyas parece haber quedado clara la inspiración en artistas como Carreño de Miranda, sin embargo nos parece muy llamativa la impronta que en su arte va a marcar el pintor contemporáneo José Moreno. Lo que no deja de sorprender un tanto si tenemos en cuenta que Moreno fue un pintor nacido en fechas muy próximas a las suyas.

\section{BIBLIOGRAFÍA}

Agulló Cobo, Mercedes (1981): Más noticias sobre pintores madrileños de los siglos XVII y XVIII, Madrid: Ayuntamiento de Madrid.

Angulo Iñíguez, Diego (1971): Pintura del siglo XVII, Ars Hispaniae, vol. XV, Madrid: Editorial Plus Ultra. Caballero Bernabé, Francisco Javier et al. (1990): "Inventario-catálogo de la pintura de Alcalá de Henares", en La Universidad de Alcalá II. Madrid: Colegio Oficial de Arquitectos de Madrid, Universidad de Alcalá de Henares, pp. 307 y 320.

Gutiérrez Pastor, Ismael (1991): “Antonio Castrejón como retratista y otras obras de su hijo Baltasar”, Anuario del Departamento de Historia y Teoría del Arte, Universidad Autónoma de Madrid, vol. III, pp. 101-108.

Gutiérrez Pastor, Ismael (2000): "Novedades de pintura madrileña del siglo XVII: Obras de José Antolínez y de Francisco Solís", Anuario del Departamento de Historia y Teoría del Arte, Universidad Autónoma de Madrid, vol. XII, pp. 75-92.

Gutiérrez Pastor, Ismael (2005): "El pintor José Moreno (c. 1630/1637-1677): revisión de su vida y nuevas obras", Anuario del Departamento de Historia y Teoría del Arte, Universidad Autónoma de Madrid, vol. XVII, pp. 67-85.

Gutiérrez Pastor, Ismael (2009): "Lienzos de José Moreno para el retablo del Santo Cristo de la Sangre de Torrijos (Toledo)", Archivo Español de Arte, 328, pp. 404-409.

Palomino y Velasco, Antonio (1947): El Museo Pictórico y Escala Óptica, Madrid: ed. Aguilar.

Pérez Sánchez, Alfonso E. (2010): Pintura Barroca en España 1600-1750, Madrid.

Pérez Sánchez, Alfonso E. (1986): Carreño, Rizi, Herrera y la pintura madrileña de su tiempo (1650-1700), cat. exp., Madrid.

Arch. esp. arte, LXXXVIII, 350, ABRIL-JUNIO 2015, 198-204

ISSN: 0004-0428, eISSN: 1988-8511, doi: 10.3989/aearte.2015.13 
Portela Sandoval, Francisco J. (1995): “Una nueva obra del pintor barroco Antonio Castejón”, Estudios de Arte. Homenaje al profesor Martín González, Valladolid: Universidad de Valladolid, pp. 561-563.

Salvador Prieto, Ma Socorro (1991): "Una Inmaculada de Antonio Castrejón, pintor del siglo XVII", V Jornadas de Arte, “Velázquez y el Arte de su tiempo”, Madrid: C.S.I.C., pp. 289-294.

Sullivan, Edward J. / Mallory, Nina A. (1982): Painting in Spain 1650-1700 from North American Collections, The Art Museum, Princeton University.

Urrea Fernández, Jesús (1975): “Obras de pintores menores madrileños: B. De Castrejón, A. Van de Pere y P. Ruíz González”, Boletín del Seminario de Estudios de Arte y Arqueología, Universidad de Valladolid, pp. 707-712.

Fecha de recepción: 19-V-2014

Fecha de aceptación: 08-X-2014

Arch. esp. arte, LXXXVIII, 350, ABRIL-JUNIO 2015, 198-204 ISSN: 0004-0428, eISSN: 1988-8511, doi: 10.3989/aearte.2015.13 